\title{
Una nueva escuela. Un nuevo modelo de dirección
}

\section{A new school. A new management model}

\author{
Emilio J. Veiga*, Fernando López-Azcárate** \\ Universidad de Santiago de Compostela *, Universidad de Santiago de Compostela**
}

\begin{abstract}
Resumen
Siguiendo propuestas y principios que encontramos reflejados en la Ley Orgánica 8/2013 para la mejora de la calidad educativa (LOMCE), nos encontramos en su artículo 122, con una idea que podemos considerar básica a la hora de la búsqueda de cambios significativos y de la procura de la mejora de la calidad en los centros educativos. Las acciones de calidad educativa partirán de una consideración integral del centro, que podrá tomar como referencia modelos de gestión reconocidos en el ámbito europeo, y habrán de contener la totalidad de las herramientas necesarias para la realización de un proyecto educativo de calidad.
\end{abstract}

Palabras claves: Liderazgo pedagógico, comunidad educativa, competencias, calidad, compromisos.

\begin{abstract}
Following proposals and principles are reflected in the Organic Law 8/2013 to improve educational quality (LOMCE), we find in Article 122, with a basic idea that we can consider when looking for significant changes and the pursuit of quality improvement in schools. Shares of educational quality will leave a comprehensive consideration of the center, which can take as reference models recognized at European level management, and must contain all the necessary for the realization of an educational project quality tools.

Keywords: Educational leadership, educational

community, competence, quality, commitments.
\end{abstract}

En esta idea de cambio los centros docentes deberán de trabajar con una planificación estratégica que precisará de un compromiso global de todos los miembros de la comunidad educativa y a su vez de un verdadero liderazgo.

Este cambio permanente es imprescindible no sólo cuando los resultados son malos, también cuando los resultados son buenos (siempre se pueden mejorar) e incluso para que sigan siendo buenos. Y este cambio ha de ser un cambio "inteligente", nacido de la autoevaluación (¿dónde estoy?) para señalar un objetivo (¿dónde quiero estar?) y alcanzar una "escuela inteligente”, una escuela que aprende y se adapta para siempre estar instalada en la excelencia.

$\mathrm{Y}$ el cambio tiene que centrarse en dos objetivos fundamentales: la mejora continua de los procesos y el cambio de la cultura organizacional, lo que precisa tanto un liderazgo pedagógico fuerte como trabajo en equipo.

El liderazgo desempeña el papel fundamental (no hay mala escuela con un buen director), lo que requiere la adquisición de las habilidades necesarias para dirigir la escuela del siglo XXI, la escuela de excelencia. Nuevos tiempos demandan nuevas competencias: inteligencia emocional (empatía y habilidades sociales) para generar equipos de trabajo eficaces y eficientes, técnicas de negociación y manejo de conflictos, capacidad de motivar y carácter para tomar las decisiones necesarias...

\section{La dirección del cambio}

Consideramos que seguimos teniendo una visión de la escuela en la que prioriza la idea de la figura del director/a como elementos referenciales. Ante esta visión podemos contrastar otra más actual, más dinámica, más participativa y en la que se centran más los estudios e investigaciones recientes sobre el liderazgo de las organizaciones escolares.

Precisamente y desde fechas bastantes recientes se está poniendo el acento y el énfasis en la idea del liderazgo compartido.

Se ha destacado que la clave está en promover la capacidad de aprendizaje de los propios agentes $\mathrm{y}$, especialmente, de los centros escolares como organizaciones, en una acción conjunta compartida (Bolívar, 2009).

Es pues una variable que de forma más o menos directa se valora como decisiva a la hora de analizar y estudiar el funcionamiento de los centros y sobre todo los cambios en la escuela de hoy.

Un creciente cuerpo de investigación sugiere que un cambio real en las escuelas requiere el desarrollo de comunidades fuertes en las que la principal labor de los equipos directivos es favorecer las mejores relaciones, el trabajo colegiado, la satisfacción profesional y en consecuencia la reflexión-acción conjunta (López-Yáñez, 2009).

En esta situación, un relevante informe de la Organización para la Cooperación y el Desarrollo Económico, OCDE (ImprovingSchoolLeadership) señala "necesitamos contrastar estas tendencias con la práctica y la forma actuales del liderazgo escolar... En muchos países hay una creciente inquietud de que la función de los directores, diseñada para las necesidades de una época diferente, pueda no ser la adecuada para enfrentar los desafíos de liderazgo que las escuelas encaran en el siglo XXI" (Pont, Nusche\&Moorman, 2008, p. 27).

El paradigma del cambio en la organización y la gestión ha de encaminarse en crear y potenciar capacidades en y entre los docentes Karen Louis (1994). Los cambios deben, así, iniciarse internamente desde dentro. Han de ser sentidos, necesitados, precisados y esto se alcanza mejor de modo colectivo, induciendo a 
los propios implicados a la mejora de su práctica, mediante un aprendizaje colegiado en sus contextos de trabajo. Si se quiere mejorar, el foco ha de ponerse en las potencialidades de la escuela y de sus elementos, sobre todo humanos.

Será en este punto en donde la dirección en quipo y el trabajo colegiado favorecerán las mejores propuestas de un liderazgo compartido y en esta línea la necesidad de una formación dirigida a los equipos más que a las personas individuales.

Todo esto ha contribuido a que la dirección pedagógica de los centros educativos se esté constituyendo, a nivel internacional y nacional, como un factor de primer orden en la mejora de la educación, al tiempo que en una prioridad de las agendas de las políticas educativas. Diversos informes internacionales lo ponen de manifiesto. Por una parte, el informe TALIS (OCDE, 2014) analiza la relevancia de un liderazgo para el aprendizaje del alumnado, del profesorado y del propio centro como organización.

\section{Dirección burocrática VS dirección compartida}

Si reparamos en el concepto de "equipo directivo" en la recientemente publicada Ley Orgánica 8/2013, de 9 de diciembre, para la mejora de la calidad educativa educativa (LOMCE), comprobamos que aparece en 15 ocasiones y principalmente se centra en destacar que dicho equipo "trabajará de forma coordinada en el desempeño de sus funciones, conforme a las instrucciones del director y las funciones específicas legalmente establecidas”, art. 131.2. Es pues esta una visión institucional muy delimitada y restrictiva que poco favorece esas visiones más internacionales que acabamos de destacar en donde la idea de dirección en equipo y con un trabajo colegiado y compartido, con verdadera implicación y búsqueda de fines comunes, favorece las propuestas que consideramos idóneas para conseguir cambios y avances significativos en las instituciones escolares. Y son las que han de servir como fuente de mejora y de progreso significativo en la institución escolar.

En una visión real de muchos centros educativos nos encontramos con unas direcciones burocratizadas hasta límites infinitos en donde los directores y los equipos directivos suelen estar sobre exigidos a nivel administrativo, no tienen tiempo para tareas de índole más pedagógica y participan poco de los procesos decisorios. La principal dificultad para consolidar un liderazgo escolar efectivo tiene relación con las tareas y actividades de los directores que han aumentado y se han complejizado en la década de los 2000. El cúmulo de las exigencias externas ha llevado en muchos casos, a una fragmentación de la función (Mulford, 2003).

Así destacamos que ser un director y ejercer el liderazgo escolar en el siglo XXI requiere el desarrollo de competencias profesionales pedagógicas complejas (Vaillant y Marcelo, 2009); y el problema es que con frecuencia, las tareas administrativas son preponderantes en detrimento de las funciones pedagógicas. Los directores dedican un alto porcentaje de su tiempo a tareas administrativas y a actividades tales como el control y la supervisión de recursos materiales y humanos (OCDE, 2009, 2012). El seguimiento y la evaluación de los progresos de aprendizaje y el desarrollo profesional de los docentes, son menos frecuentes (UNESCO, 2008; Talis, 2015).

Señalamos al respecto, el informe de la OECD TeacherEvaluation in Chile OECD Reviews of Evaluation and assessment in education (2013), que en relación al rol que los directivos desempeñan destaca que:

"No obstante, es habitual que los directores de los colegios estén sobrepasados con la labores que deben realizar en los establecimientos y, por lo general, parecen no tener el tiempo para participar adecuadamente en las labores de orientación, monitoreo y evaluación de los profesores. Por ejemplo, las observaciones de aula efectuadas por los directores parecieran ser relativamente ocasionales. En general, no existe un mecanismo para garantizar que cada uno de los profesores reciba una retroalimentación profesional adecuada."

Murillo (2005), por su parte, elaboraron una investigación en la que estudiaron cómo reparten su tiempo los directivos de centros docentes de Primaria en España, qué diferencias se dan en ese reparto en función de diversas variables contextuales y cómo influye todo ello en la dinámica del centro. Entre sus resultados más relevantes destaca que los directivos dedican el 37\% del tiempo a tareas administrativas, el $27 \%$ a tareas relacionadas con el liderazgo pedagógico, el $16 \%$ a tareas ligadas al contacto con las familias, el $15 \%$ a tareas que tienen que ver con su desarrollo personal y el $6 \%$ a otras actividades.

Cabría también destacar la Investigación Iberoamericana sobre Eficacia Escolar, analizando el trabajo de los directivos de 91 escuelas de ocho países de la región (Murillo, 2007), encontró que los directivos de la zona dedican la mayor parte del tiempo a tareas burocráticas (36,4\%), seguidas de actividades de liderazgo pedagógico (20,1\%); a continuación invierten tiempo en su desarrollo profesional $(17,3 \%)$ y en las familias $(16,1 \%)$

$\mathrm{Y}$ en contraste con estos datos en una reciente encuesta solicitada a los profesionales de la dirección de centros educativos de Galicia, formulada por la Agencia para la Modernización Tecnológica de Galicia (AMTEGA) se cuestionaba sobre cuáles eran los tiempos de gestión burocrática, de relación, de uso en las Tics, y de liderazgo pedagógico, entre otros. Pendiente de la publicación de los resultados de dicha encuesta la sensación generalizada entre muchos profesionales que están ejerciendo en las direcciones escolares, en las jefaturas de estudio y en las secretarías de los centros era de que una parte muy considerable de su tiempo de dedicación a las tareas organizativo-directivas se empleaba en dimensiones como la atención a las múltiples aplicaciones informáticas que hoy en día hay que atender a diario - destacamos la existencia de más de 15 aplicativos de gestión diaria con el uso de las Tics que demandan la atención de los equipos directivos en los centros escolares gallegos-, la atención a los procesos y documentos que se generan de forma periódica a lo largo 
de un curso escolar: cumplimentación de estadísticas del centro, inicio escolar, comedores, transporte, períodos de reserva de plazas escolares, matriculaciones, gestión de libros, evaluaciones de sistema...

"cada vez tenemos más máquinas y aplicaciones informáticas que supuestamente nos deberían facilitar las cosas y cada vez se originan y mueven más papeles...vivimos en el mundo del papel no productivo”.

Los directores suelen estar sobre exigidos a nivel administrativo, no tienen tiempo para tareas de índole más pedagógica y participan poco de los procesos decisorios. La principal dificultad para consolidar un liderazgo escolar efectivo tiene relación con las tareas y actividades de los directores que han aumentado y se han complejizado en la década de los 2000. El cúmulo de las exigencias externas ha llevado en muchos casos, a una fragmentación de la función (Mulford, 2003).

En conclusión podemos a confirmar la relación encontrada en los citados estudios nacionales e internacionales, y en los reseñados en el ámbito gallego en donde se aprecian una relación estadísticamente significativa entre el tiempo que los directivos dedican a tareas relacionadas con la gestión burocrática y su insatisfacción con el rol directivo y por contra la relación directa entre el liderazgo pedagógico y un mejor desempeño de los estudiantes de la escuela, así como entre dicha dedicación y una satisfacción mayor de los docentes con la dirección.

Llegados a este punto destacamos Informes internacionales sobre liderazgo OCDE (2009) que en su prefacio afirma que "el liderazgo escolar ha de ser una prioridad política en todo el mundo. Una mayor autonomía y una mayor concentración en los resultados educativos y escolares ha llevado a que sea esencial reconsiderar la función de los líderes de los centros escolares".

En estudios de la propia OCDE y de varios ministerios de educación de países europeos y del ámbito internacional, Iberoamérica, Australia, Nueva Zelanda entre el 2006 y el 2009 identifican y destacan unas áreas de acción que, vistas y analizadas en su conjunto, pueden y han de ayudar a mejorar la práctica del verdadero liderazgo escolar. $\mathrm{Y}$ todas ellas ponen el punto de inflexión en la necesidad de:

- Redefinir las responsabilidades del líder escolar, centrando los esfuerzos en funciones que ayuden a la mejora de los resultados escolares, último fin de las políticas educativas.

- Distribuir el liderazgo escolar, comprometiendo y reconociendo una participación más amplia en los equipos de liderazgo y en las potencialidades humanas de los mismos.

- Desarrollar habilidades para un liderazgo escolar eficaz a lo largo de diferentes etapas de la gestión y de la práctica.

- Hacer del liderazgo escolar un ámbito profesional más activo, y así mismo más atractivo al garantizar reconocimiento económico y perspectiva de carrera profesional apropiados (no puede ser que los tiempos de atención a la dirección de los centros en la Comunidad Autónoma de Galicia estén regulados por normativa de los años 1997, O. de 22 de julio de 1997 por la que se regulan determinados aspectos de organización y funcionamiento de las escuelas de educación infantil, de los colegios de educación primaria y de los colegios de educación infantil y primaria dependientes de la Consellería de Educación y Ordenación Universitaria. O la O. do 1 de agosto de 1997 por la que se dictan instrucciones para el desenvolvimiento do Decreto 324/1996 polo que se aprueba o Reglamento orgánico dos institutos de educación secundaria e se establece su organización e funcionamiento)

El citado estudio como hemos dicho, fruto del trabajo conjunto de varios países de la OCDE, de expertos y organizaciones internacionales (la Unión Europea, la Comisión o la International Principales Association) y de representantes de distintas organizaciones sindicales, fue concebido para apoyar el diseño y la implementación de políticas de liderazgo escolar dirigidas a la mejora de la enseñanza y el aprendizaje. En él han colaborado 22 países,

Algunos de ellos miembros de la Unión Europea (países nórdicos, España, Portugal, Suecia, Reino Unido, etc.) y otros (como Corea, Nueva Zelanda, Australia, Israel o Chile) ajenos a ella; países que, tomados en su conjunto, ofrecen un marco comparativo de considerable extensión. Pont, B.; Nusche, D. y Moorman, H. (2010).

Algunas de las principales dimensiones fruto de estas investigaciones las reflejamos a continuación.

\section{$\operatorname{Re}($ definición) de las responsabilidades del liderazgo escolar}

Entendemos por "liderazgo", fundamentalmente, la capacidad de ejercer influencia sobre otras personas, de manera que éstas puedan tomar las líneas propuestas como premisa para su acción. Esta influencia, no basada en el poder o autoridad formal, se puede ejercer en distintas dimensiones, especialmente en el plano organizativo, cuando una dirección logra alcanzar consenso y moviliza a la organización en torno a metas comunes (Leithwood, Day, Sammons, Harris y Hopkins, 2006)

Se trata, por un lado, de dar mayor autonomía y, por otro, de acompañarla del apoyo necesario para poder ejercerla. ¿De qué sirve insistir en la autonomía si no se ofrecen ni el apoyo ni los instrumentos para poder desarrollarla? Véase la realidad social, y en consecuencia las configuraciones familiares que se pueden encontrar hoy en día en las escuelas en relación al modelo que se reflejaba hacen veinte años atrás y seguimos a trabajar con herramientas y normativas de eses años.

Es necesario asegurarse de que las responsabilidades estén bien definidas y se centren en la mejora de los resultados escolares; una mejora que claramente se produce cuando los directores y directoras se dedican principalmente a apoyar, evaluar y desarrollar al profesorado, a crear y potenciar equipos que trabajen conjuntamente hacia esa mejora.

Un liderazgo que se encamine no solo a la mejora de los resultados escolares, sino que trabaje en conjunto y se apoye mutuamente para favorecer la mejora del sistema en general, evitando así el aislamiento que puede sufrir la dirección cuando se mantiene exclusivamente dentro de 
su escuela, dentro del espacio protegido "el área de gestión”.

\section{Coparticipación}

Tal y como indicábamos anteriormente la realidad de la dirección escolar es que las responsabilidades son muchas y no pueden recaer sobre una única persona. Es importante concebir la dirección como un equipo donde se distribuyan las responsabilidades. La distribución del liderazgo juega un papel decisivo a la hora de planificar la sucesión con líderes de calidad.

Liderazgo compartido o distribuido, como un marcado estilo diferenciador muy adecuado a la función directiva moderna, actual. Y en consecuencias debemos destacar la idea de la necesidad de la búsqueda, adquisición y desarrollo de habilidades específicas, determinadas, variadas, lo que justificará siempre la delegación y distribución de tareas entre los miembros del equipo directivo y de cualquier otro equipo que se conforme cara al logro de la mejora educativa en un determinado centro. Una dirección con estilo compartido logrará siempre de la comunidad escolar: mayor implicación, mayor compromiso, mayor participación, mayor satisfacción.

A la hora de hablar de copartición, de compromiso colegiado no debamos olvidar la tan necesaria recuperación de los Consejos Escolares como órganos de vital importancia que nunca históricamente han desarrollado correctamente sus funciones (Enguita, 1993; San Fabián, 2006; Bolívar, 2010)

Pensamos que en el tema de la distribución de responsabilidades ocupa un lugar esencial el consejo escolar, órgano este bastante abandonado hasta el momento. De él forman parte voluntarios que es probable que carezcan de una formación apropiada y de incentivos para ejercer las tareas que se les encomienda. Resulta vital aclarar la función de dichos consejos, crear nuevos incentivos para sus miembros e impartirles la formación que precisen. También sería conveniente seleccionar a sus miembros de acuerdo con las necesidades particulares de cada escuela y de cada consejo escolar. En diversos países europeos se imparte formación específica dirigida a los miembros de los consejos escolares con resultados bastante positivos.

\section{Formación competencial a lo largo de la carrera profesional}

Para dotar a los directores de las nuevas habilidades y competencias requeridas por el liderazgo en la organización y en la gestión del centro educativos esta tercera dimensión de la formación resulta de vital importancia. Y sobre todo en un sistema tan hibrido como el tradicionalmente implantado en España en donde, en línea contraria a otros muchos países de la OCDE, y de otros ámbitos internacionales, el principal requisito que se les exige a los candidatos a la dirección es el de proceder de las filas del profesorado. Y la verdad no debemos en ningún momento perder de vista que el profesorado se ha formado fundamentalmente para enseñar, pero no para gestionar personal o presupuestos, ni para responder a las múltiples variables en las que se encuentran inmersos en la sociedad de hoy los equipos directivos. Por ello es importante una formación inicial del candidato centrado en las habilidades necesarias para el ejercicio de la dirección, así como una formación continua que acompañe al equipo directivo en todas las etapas de su trayectoria.

\section{El atractivo del liderazgo escolar}

Figura en las conclusiones de todos los estudios al respecto y resaltado con letras remarcadas la recomendación a los gobiernos y responsables globales internacionales que deben de llevar a cabo políticas de reconocimiento que hagan más atractiva la función directiva. Como comentábamos con anterioridad en España ningún gobierno ni ningún personaje con responsabilidades educativas ha cambiado jamás, fuera del color que fuera, el modelo de dirección para encaminarlo hacia una dirección profesionalizada. Así acontece que en las últimas décadas las administraciones educativas se han visto muy forzadas a realizar nombramientos debido a la falta de candidatos que principalmente por la falta de indefinición de lo que $\mathrm{s}$ eles brinda y del futuro que le augura apuestan por dar el salto a asumir las direcciones escolares, e incluso solo formar parte de los equipos directivos y de gestión.

Por supuesto y en consecuencia de todo lo citado nos encontramos con muy poca falta de sensibilidad institucional hacia estés equipos directivos en cuento a los aspectos económicos, en cuento a los horarios de dedicación, y sobre todo en cuanto a las pocas salidas que ofrece a largo plazo.

\section{Discusión y Conclusiones}

¿Cómo estamos en verdad?; ¿Tenemos margen de mejora? ¿Qué hacer?...estas y otras muchas cuestiones que se nos pueden y de hecho se nos plantean debemos abordarlas de forma reflexiva, serena, colegiada, con fundamentación real y con espíritu socio-crítico. Como cita Nélida Zaitegui, quién tiene preguntas busca respuestas y quién tiene un "por qué” busca un “cómo”.

Destaquemos algunas consideraciones a modo de resumen que pueda abrir el debate y nos lleve a la procura de soluciones verdaderas.

- El objetivo prioritario de las políticas educacionales en el siglo XXI debe ser garantizar a todos los estudiantes los aprendizajes imprescindibles que les que posibiliten, sin riesgo de exclusión, la integración y participación activa en la vida pública. El liderazgo en la enseñanza está, sin duda, para hacerlo posible. Necesitamos, pues, los mejores equipos directivos que puedan ejercer un liderazgo educativo. Para esto, en primer lugar, como sugiere el informe de la OCDE (Pont et al., 2008), se requiere hacer de la dirección escolar una profesión atractiva. Atraer a los mejores candidatos deben potenciarse en remuneraciones, carrera profesional y formación.

- Centremos los esfuerzos en la procura de una formación inicial y en servicio adecuadas. Los líderes escolares necesitan capacitación específica para responder al aumento de funciones y responsabilidades, en particular sobre estrategias para mejorar los resultados escolares. 
- La nueva gestión enfocada al gobierno de la organización de los centros está demandando un cambio de papel que, justamente, al no haberse producido una reestructuración organizativa, lo impiden. De ahí la necesidad de un liderazgo de la dirección que incite, de un modo "transformativo", al desarrollo del establecimiento escolar como organización.

- La visión del verdadero liderazgo ha de ir unido a iniciativas e influencias en donde la corresponsabilidad este distribuida entre todos los miembros (liderazgo distribuido) de la escuela. Desde las “organizaciones que aprenden" se subraya la necesidad de distribuir o dispersar las tareas dinamizadoras en el conjunto del profesorado, como hemos comentado en otro lugar (Bolívar, 2000)

En resumen de lo que en verdad se trata, en último extremo, es de cómo garantizar a toda la sociedad y más concretamente al elemento básico del sistema escolar "el alumnado" sus derechos de obtener los mejores resultados y la mejor de las formaciones para la vida, su vida y para ello no basta tener algunas buenas escuelas que funcionan bien, sino hacer de cada escuela una gran escuela. El liderazgo en la enseñanza ocupa, sin duda, un lugar en dicha tarea.

\section{Referencias}

Álvarez Manuel (2010). Liderazgo compartido. Buenas prácticas de dirección escolar. WoltersKluwer

Bolívar, A. (2000). Los centros educativos como organizaciones que aprenden. Madrid: La Muralla.

Bolívar, A. (2009). La autonomía en la gestión como nuevo modo de regulación. Espacios en Blanco, 19, 35-68.

Decreto 374/96, de 17 de octubre, por el que se aprueba el reglamento orgánico de las escuelas de educación infantil y de los colegios de educación primaria de Galicia.

Decreto 324/1996, do 26 de julio, por el que se aprueba el Reglamento orgánico de los institutos de educación secundaria de Galicia.

Fernández Enguita, M. (1993). La profesión docente y la comunidad escolar: crónica de un desencuentro. Madrid, Morata/Paideia.

Fullan, M. (2002). El significado del Cambio educativo, un cuarto de siglo de aprendizaje. Revista de Currículum y Formación del Profesorado, n. 6, 1-2.
Fullan, M. (2010). The moral imperative realized. Thousand Oaks: Corwin

Ley Orgánica 2/2006, de 3 de mayo, de educación. (B.O.E de 4 de mayo de 2006)

Ley orgánica 8/2013, de 9 de diciembre, para la mejora de la calidad educativa (B.O.E de 10 de decembro de 2013)

Louis, K.S. (1994). Beyond Managed Change: Rethinking how Schools Improve. School Effectiveness and Improvement, 5 (1), 2-24.

Mulford, B. (2003). School Leaders: Changing roles and impact on teacher and school effectiveness. Paris: OCDE

Murillo, F. J. (2005). La investigación sobre eficacia escolar. Barcelona: Octaedro.

Murillo, F. J. (2006). Una dirección escolar para el cambio: del liderazgo transformacional al liderazgo distribuido. Revista Electrónica Iberoamericana sobre Calidad, Eficacia y Cambio en Educación.

OECD (2012). Delivering School Transparency in Australia: National Reporting through My School.

OECD (2013). Leadership for 21st Century Learning,

Educational Research and Innovation, OECD Publishing

Pont, B (2010). Liderazgo y autonomía del centro escolar: perspectivas internacionales. Directora del Proyecto "Mejorando el liderazgo educativo" 1. Dirección General de Educación - OCDE

Santos, M (2002). La Escuela que aprende. Madrid: Morata. UNICEF (2004). ¿Quien dijo que no se puede?. Escuelas efectivas en sectores de pobreza. Santiago de Chile: Unicef, Mineduc.

San Fabián Maroto, J. L. (2006): “Consejos Escolares: renovarse o morir”. En Organización y Gestión Educativa, $n^{\circ} 1$, enero- febrero: $\mathrm{p} 38$

VV. AA. (2000) "Democracia, educación y participación en las instituciones educativas”. En Revista Kikirikí, no 55/56 (monográfico), pp. 35-122, M.C.E.P. Morón (Sevilla).

Zaitegi, N. El equipo directivo y las gestión de la convivencia en el centro. Revista Perspectiva. Junta de Andalucía (2006)

Welch, A.. (2011). PISA Performance and AustralianEducation: Myths and Realities. Revista Española de Educación Comparada, , 18 (Special), 89-124 